\title{
Chaos with a high-dimensional torus
}

\author{
Jumpei F. Yamagishi $\odot$ and Kunihiko Kaneko ๑* \\ Graduate School of Arts and Sciences, The University of Tokyo, 3-8-1 Komaba, Meguro-ku, Tokyo 153-8902, Japan
}

(Received 20 August 2019; accepted 16 March 2020; published 15 April 2020)

\begin{abstract}
Transition from quasiperiodicity with many frequencies (i.e., a high-dimensional torus) to chaos is studied by using $N$-dimensional globally coupled circle maps. First, the existence of $N$-dimensional tori with $N \geqslant 3$ is confirmed while they become exponentially rare with $N$. Besides, chaos exists even when the map is invertible, and such chaos has more null Lyapunov exponents as $N$ increases. This unusual form of "chaos coexisting with a torus" exhibits delocalization and slow dynamics of the first Lyapunov vector. Fractalization of tori (i.e., strange nonchaotic attractor) at the transition to chaos is also suggested. These results integrate the classic viewpoints on turbulent behavior as high-dimensional torus by Landau and as low-dimensional chaos by Ruelle and Takens.
\end{abstract}

DOI: 10.1103/PhysRevResearch.2.023044

Quasiperiodic motion with multiple incommensurate frequencies is ubiquitously observed in nature [1-5] including hydrodynamics [6,7], semiconductor lasers [8], electric circuits [9], chemical reactions [10], heart rhythm [11], and the brain [12], while bifurcation(s) from quasiperiodicity to chaos appears in general as a parameter of the system in concern is changed.

This transition from periodic or quasiperiodic motions to chaos gathered much attention in the 1970s and 80s, where several routes to chaos were unveiled in theory, simulations, and experiments $[1-4,13]$. They include a perioddoubling route to chaos with its renormalization-group analysis [14] and intermittency [15], as well as the transition from quasiperiodicity (on a torus attractor) to chaos [16,17]. For this last case, frequency lockings with devil's staircase [18] and doubling or fractalization of tori [19] are uncovered in regard to two-dimensional tori.

Transition from a torus with more than two frequencies to chaos, in contrast, is not fully explored, in spite of its historical significance in the investigation of fluid turbulence. Landau proposed to understand turbulence as (possibly an infinite number of) successive Hopf bifurcations leading to a torus with an infinite number of (incommensurate) frequencies [20]. In contrast, it was noted that at any vicinity of a dynamical system having a three-dimensional-torus attractor, there can appear chaotic motion following some structural perturbations to the dynamical system [21-23]. This theorem, on the one hand, promoted the experimental studies on the onset of turbulence by low-dimensional chaos [1,7]; on the other hand, it was sometimes overestimated and misinterpreted as tori of higher than two dimensions being easily destabilized and replaced by chaos with most slight structural modifications

\footnotetext{
*kaneko@complex.c.u-tokyo.ac.jp

Published by the American Physical Society under the terms of the Creative Commons Attribution 4.0 International license. Further distribution of this work must maintain attribution to the author(s) and the published article's title, journal citation, and DOI.
}

(i.e., by adding any nonlinear term). The latter, indeed, is not the case: By taking dynamical systems with three or more frequencies, high-dimensional torus attractors are often observed. Then, consider changes in the parameter values for dynamical systems with a high-dimensional torus attractor and compute the fraction of parameter values for which chaos attractor appears. Such a fraction is important to discuss how frequent quasiperiodic or chaotic motion is in nature, but it is not obtained according to the study by Ruelle and Takens.

With subsequent numerical and experimental studies, the existence of three- or four-dimensional torus attractors has been confirmed [7,24-26], where structural perturbations to such dynamical systems often lead to lower-dimensional tori due to frequency lockings, rather than chaotic attractors. However, of note is that chaos can appear even for weak nonlinearity, albeit in a small fraction. This is in contrast to the case with two-dimensional tori for which sufficiently strong nonlinearity is necessary for chaos to appear. Among others, Baesens et al. investigated the behavior of chaos near a three-dimensional torus in depth [26] with detailed bifurcation analysis for two-dimensional torus maps and reported the finding of toroidal chaos (see also Refs. [27,28]). Still, the nature of chaos near high-dimensional tori has not been fully explored.

In this paper, we explore this long-lasting problem of transition to chaos from high-dimensional torus attractors. We mainly address three questions. The first question is with regard to the existence of high-dimensional tori-Can they still exist as attractors with the increase in dimension of tori, i.e., the number of incommensurate frequencies? We will present how the fractions of tori and chaos in the parameter space depend on the dimensionality and nonlinearity of the system.

The second question is with regard to the characteristics of chaos that emerges from a high-dimensional torus. To be specific, we report the finding of chaos coexisting with a high-dimensional torus, as characterized by a large number of null Lyapunov exponents, accompanied by one or a few positive and some negative exponents. The eigenvector for the maximum positive exponent is extended over degrees 

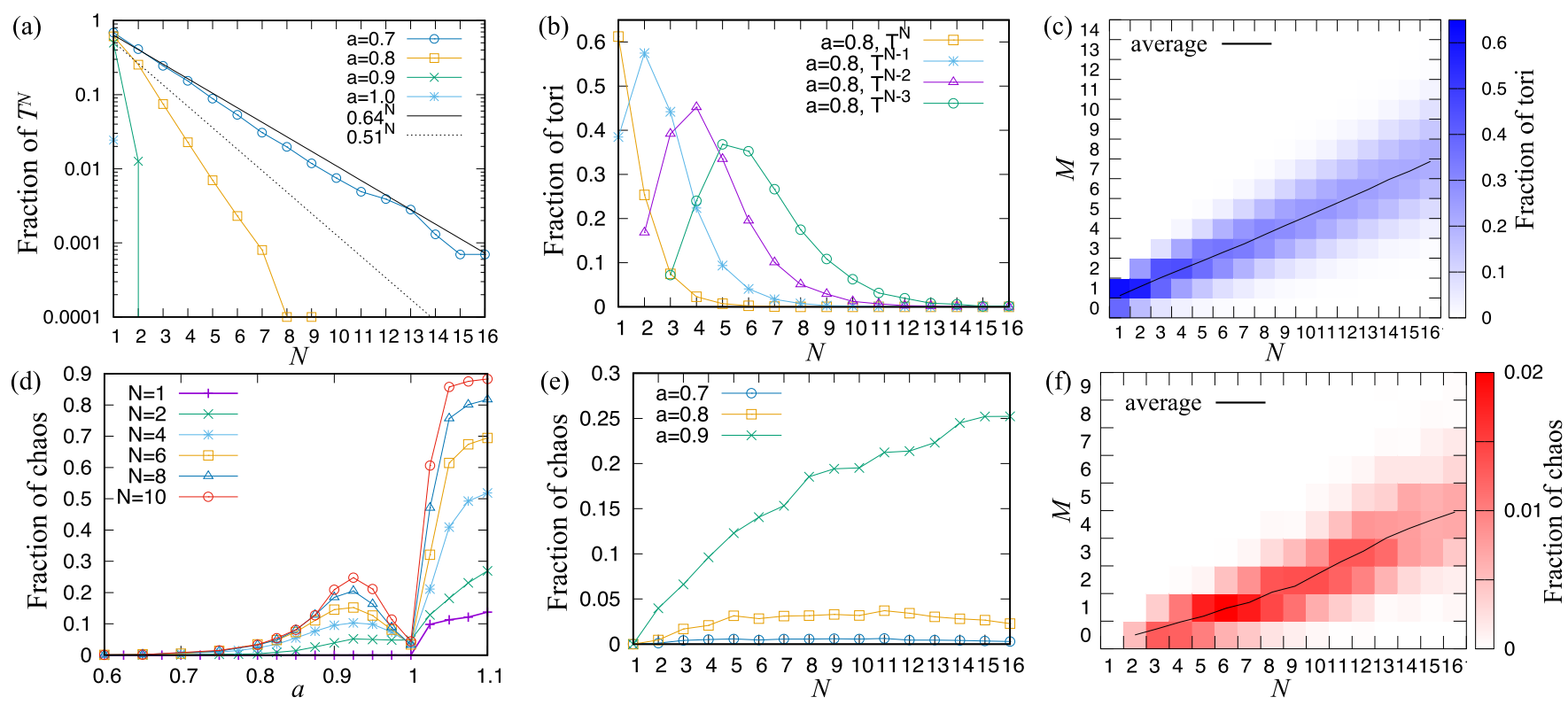

FIG. 1. Fractions of tori and chaos in map (1) over a random choice of natural frequencies $\vec{\Omega}$. The coupling constant $J_{i j}=b$ is set to 0.1 , while $10^{4}$ samples of $\vec{\Omega}$ are randomly chosen as $\Omega_{i} \in\{0.0005,0.001, \cdots, 0.5\}$. (see also Supplemental Material [30], Fig. S1). (a) Dependence of the fraction of $T^{N}$ on $N$. (b) Dependence of the fractions of $T^{N}, T^{N-1}, T^{N-2}$, and $T^{N-3}$ on $N$. (c) Fractions of $M$-dimensional tori for $a=0.8$. (d) Dependence of the fraction of chaos on $a$ and $N$. The critical value $a_{c}$ to lose invertibility is 1 for $N=1$, and $>0.9$ for $N \geqslant 2$ : e.g., $a_{c}(2,0.1) \simeq 0.91$ and $a_{c}(4,0.1) \simeq 0.94$. (e) Dependence of the fraction of chaos on $N$. (f) Fractions of chaos with an $M$-dimensional torus for $a=0.8$.

of freedom and shows slow itinerant motion with the $1 / f^{v}$ spectrum, suggesting characteristics of chaos with a highdimensional torus.

The third question to be touched upon is the nature of how a torus is destabilized, leading to chaos with a torus. To be specific, we study the following coupled circle map:

$$
\begin{aligned}
x_{i}(t+1)= & x_{i}(t)+\Omega_{i}+\frac{a}{2 \pi}\left[\sin \left(2 \pi x_{i}(t)\right)\right. \\
& \left.+\frac{1}{N-1} \sum_{j=1 ; j \neq i}^{N} J_{i j} f\left(x_{i}(t), x_{j}(t)\right)\right],
\end{aligned}
$$

where $N$ oscillators are heterogeneous (i.e., have different natural frequencies $\Omega_{i}$ ) and globally coupled [29]. Here, we mainly consider the model with $J_{i j}=b$ and $f\left(x_{i}(t), x_{j}(t)\right)=$ $\sin \left(2 \pi x_{j}(t)\right)$, although the results below do not depend on the precise form of them (see Supplemental Material [30], Sec. S1).

The map is an extension of globally coupled map to include heterogeneity over elements, and a discrete-time version of coupled oscillators [5,31]. It can be generally regarded as a map on the oscillation phases for Poincaré map of a flow obeying some ordinary differential equation. Hence, the attractors with $M$ incommensurate frequencies (i.e., with $M$ null Lyapunov exponents, without positive ones) correspond to $(M+1)$-dimensional tori in the continuous-time flow system [32]. Another important feature of map (1) is that when $a<{ }^{\exists} a_{c}(N, b)$, the Jacobian is always nonzero, and thus the dynamical system is invertible [34]. For example, $a(1+b)<$ 1 is a sufficient condition for the invertibility in the case of $J_{i j}=b$.
The case with $N=1$ was first introduced as a (sine)-circle map by Arnold for the study of two-dimensional tori, i.e., as a map on one phase of oscillation sampled by the inverse of the other frequency. In this case, the map is shown to be invertible for $a<a_{c}(1, b)=1$ where the attractor is either a torus or a periodic cycle; whereas, for $a>1$, it is either chaos or a periodic cycle. This map has been investigated as a standard model for the transition to chaos (at $a=a_{c}$ ) from quasiperiodicity on a two-dimensional torus [16-19]. In contrast, for $N=2$, which corresponds to the map for a threedimensional flow, chaos can appear even for $a<a_{c}(2, b)$ [24-26].

To examine the nature of each attractor, we computed the $N$-dimensional Lyapunov spectra by iterating map (1) $2.5 \times 10^{6}$ times after dropping the initial $5 \times 10^{5}$ times of iterations from uniformly-random initial conditions $x_{i}(0)$. The Lyapunov exponent is regarded as zero if its value is less than $10^{-4}$ and larger than $-10^{-5}[35]$.

First, the fractions of tori and chaos were numerically computed, by randomly choosing the natural frequencies $\vec{\Omega}$. As shown in Fig. 1(a), even the highest-dimensional (i.e., $N$-dimensional in map or $(N+1)$-dimensional in flow) tori exist, while their fraction decreases exponentially with $N$. This decrease is mostly due to locking to lower-dimensional tori, rather than due to replacement by chaos [Figs. 1(b) and $1(\mathrm{c})]$. As a result, the average dimensionality of torus attractors linearly increases with $N$ and its saturation with the increase in $N$ is not observed [Fig. 1(c)]. It suggests that high-dimensional [i.e., $O(N)$-dimensional] tori considerably exist, even for $N \geqslant 3$.

The exponential decrease in the highest-dimensional tori against $N$ can be understood as follows. Consider a dynamical system consisting of $N$ modes with incommensurate 

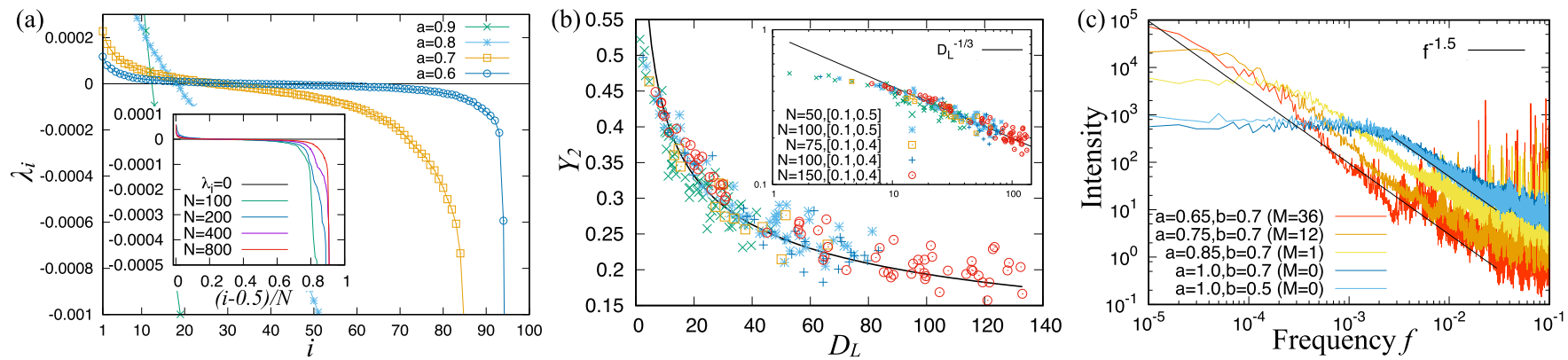

FIG. 2. Properties of chaos with a torus. (a) Lyapunov spectra $\vec{\lambda}$ with $N=100$ and $b=0.7$. Different colors correspond to different nonlinearity $a$, while natural frequencies $\vec{\Omega}$ are uniformly distributed in $(0.1,0.5]$. (inset) the scaled plot with $a=0.65$ and $b=0.5: \lambda_{i}$ vs $(i-0.5) / N$ for $N=100,200,400,800$. (b) The inverse participation ratio of the first Lyapunov vector $Y_{2}$ vs Lyapunov dimension $D_{L}$. $Y_{2}$ is calculated as the average over $2.5 \times 10^{6}$ steps for chaotic attractors with different parameters of $a=0.53 \sim 0.98, b=0.1 \sim 0.9$, and $N=50,75,100,150 . \vec{\Omega}$ are uniformly distributed in $(0.1,0.5]$ or $(0.1,0.4]$. The black line shows the curve $Y_{2} \propto D_{L}^{-1 / 3}$. (inset) the log-log plot. (c) Power spectra of the first Lyapunov vector for chaos with an $M$-dimensional torus (red: $a=0.65, b=0.7, M=36$; orange: $a=$ $0.75, b=0.7, M=12$; yellow: $a=0.85, b=0.7, M=1$ ) and standard chaos (i.e., $M=0$; blue: $a=1.0, b=0.7 ;$ cyan: $a=1.0, b=0.5$ ) with $N=100$. The slopes of the black lines are -1.5 .

frequencies. If the modes do not (or little) interact with each other, an $\mathrm{N}$-dimensional torus will exist as an attractor. Now, consider the addition of another mode that has a certain interaction with the other modes: The ratio of its frequency with those of the pre-existing modes may be close to a rational value, which leads to locking to a lower-dimensional torus. As a simple rough estimate, let us assume that such locking occurs with a certain probability, $p$. Then, the probability that no locking occurs is estimated as $(1-p)^{N}$. This gives a rudimentary understanding of why the fraction of $N$-dimensional tori decreases exponentially [36].

In the case with $N \geqslant 2$, chaos can appear even in the invertible regime, in contrast to the case with $N=1$ in which chaos can exist only in the noninvertible regime $a>a_{c}$ [Figs. 1(d)-1(f)]. Following Ruelle-Takens-Newhouse [21,22] (and earlier studies cited in Ref. [21]), chaos can appear from a (high-dimensional) torus even in the weak nonlinearity (i.e., invertible) regime, $a<a_{c}$. Here, in the noninvertible regime, the fraction of complete locking to fixed points (rather than chaos) increases near $a=1$, as in the case with $N=1$ [see Fig. 1(d) and Supplemental Material [30], Fig. S2]. Figures 1(d) and 1(e) reveal that the fraction of chaos increases with $N$, while the increase is saturated in the invertible regime.

Remarkably, chaos in the invertible regime often has not only positive Lyapunov exponent(s) but also multiple null Lyapunov exponents [Fig. 1(f)], in contrast to that in the noninvertible regime. In other words, such chaos coexists with a torus. Hence, we term this form of chaos with $M$ null Lyapunov exponents as chaos with an $M$-dimensional torus (or, with an $(M+1)$-dimensional torus in flow).

Note that, in the invertible regime, $\sum_{i=1}^{N} \lambda_{i} \leqslant 0$ always holds because the volume of attractors cannot increase locally due to dissipation, as is also confirmed numerically. Thus, chaos with a torus cannot appear for two-dimensional maps (which correspond to three-dimensional flows studied earlier following the line of Ruelle-Takens scenario). The appearrance of chaos with a torus is possible only for the map with $N \geqslant 3$ (i.e., $\geqslant 4$-dimensional flows) that allows for the Lyapunov spectrum $(+,+, \cdots, 0,0, \cdots,-,-, \cdots)$ [see Fig. 1(f)]. Then, how many null Lyapunov exponents can chaos with a torus have in a high-dimensional system? To address this question, we studied the map (1) by increasing $N$ to $\geqslant 50$, where the natural frequencies $\vec{\Omega}$ are uniformly distributed in an interval. Figure 2(a) shows the Lyapunov spectra $\vec{\lambda}$ for different $N$ plotted by scaling the index as $(i-0.5) / N$. It suggests that the torus dimension $M$ is an extensive variable, proportional to $N$. Indeed, this is consistent with the result in Fig. 1(f) that the average number of neutral Lyapunov modes in chaos with a torus increases linearly with $N$. Chaos with a torus shows the accumulation of $O(N)$ null Lyapunov exponents [38].

Now, the characteristic behaviors of chaos with an $M$ dimensional torus will be studied. To examine whether chaotic motion spreads over $M$-dimensional space or is localized, we computed the behavior of the first Lyapunov vector $\vec{v}^{(1)}$. First, the localization of the "chaos mode" is quantified by its inverse participation ratio, $Y_{2} \equiv\left\langle\sum_{i=1}^{N}\left|v_{i}^{(1)}\right|^{4}\right\rangle_{t}$; if $\vec{v}^{(1)}$ is extended to all $N$ elements, $Y_{2}$ is $O(1 / N)$, and if the vector is localized, $Y_{2}$ is $O(1)$ [39-41]. Figure 2(b) shows the negative correlation between $Y_{2}$ and the Lyapunov dimension $D_{L}$, where $Y_{2}$ is approximately proportional to $1 / D_{L}^{\alpha}$ with the exponent $\alpha \approx 1 / 3$, independent of the parameters $N, \vec{\Omega}, a, b$. Here, note that the Lyapunov dimension of chaos with an $M$-dimensional torus is at least $M+1$, and thus $D_{L}$ approximately quantifies its torus dimension $M$ in the invertible regime [42]. For standard chaos in high-dimensional dynamical systems, $Y_{2}$ is $O(1)$ against the increase in the dimension $N$, i.e., the Lyapunov vector for the chaos mode is localized. In contrast, $Y_{2}$ for chaos with an $M$-dimensional torus is decreased against $M$ (and $D_{L}$ ) with $\alpha \approx 1 / 3<1$, which suggests that the spreading of the chaos mode over elements (oscillators) $i$ is partial, compared with the fully extended case.

To see the nature of this localization, the temporal event of $\vec{v}^{(1)}$ is plotted in Supplemental Material [30], Fig. S4. This suggests partial spreading of the vector, as well as its slow itinerant motion. We then computed the power spectra of the first Lyapunov vector $\vec{v}^{(1)}$. As shown in Fig. 2(c), $1 / f^{v}$ spectra are observed for chaos with a torus, with the exponent $v \approx 3 / 2$, over a wide range of frequencies. As the 

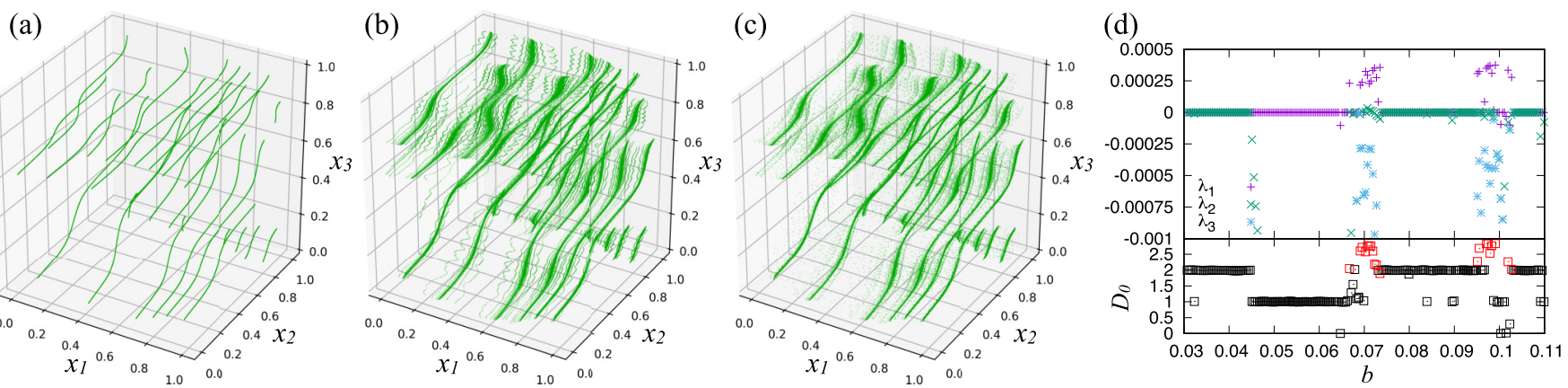

FIG. 3. Bifurcation in map (1) with the change of coupling constant $b$ for $N=3, a=0.7, \vec{\Omega}=(0.198,0.4795,0.1945)$. (a)(c) $\left(x_{1}(t), x_{2}(t), x_{3}(t)\right)$ are plotted for $2 \times 10^{5}<t \leqslant 4 \times 10^{5}$. (a) $b=0.06$ (1-torus). $\vec{\lambda}=\left(5 \times 10^{-9},-0.002,-0.01\right), D_{0} \simeq 1.0$, and $D_{L}=1$. (b) $b=0.06675$ (fractal 1-torus). $\vec{\lambda}=\left(1 \times 10^{-8},-0.0002,-0.002\right), D_{0} \simeq 1.5$, and $D_{L}=1$. (c) $b=0.0667$ (chaos). $\vec{\lambda}=(0.0002,4 \times$ $10^{-7},-0.002$ ), $D_{0} \simeq 2.05$, and $D_{L} \simeq 2.1$. (d) Dependence of Lyapunov exponents $\lambda_{i}$ (top) and box counting dimension $D_{0}$ (bottom) on $b$. The box counting dimensions for chaos (torus), computed for $240^{3}$ bins, are shown in red (black) symbols. Clear deviations of $D_{0}$ from 0 , 1 , or 2 for black symbols correspond to fractal-torus attractors.

torus dimension increases, $1 / f^{v}$ behavior is extended to much lower frequencies.

Lastly, the transition from a torus to chaos is studied by using the case with $N=3$. In Figs. 3(a)-3(c), examples of torus and chaos with a torus are shown (see also Supplemental Material [30], Figs. S5 and S6 for other examples). As depicted in Fig. 3(d), the dimensionality (i.e., box counting dimension $D_{0}$ ) of a torus first decreases with the increase in the coupling constant $b$, and at some value of $b$, chaos appears "on the torus." In the vicinity of the transition to chaos with a torus, the curve of torus attractor waves down to a smaller scale, and by magnifying it the wavy structure persists: In other words, strange nonchaotic (or, fractal-torus) attractors with noninteger dimensions exist [see Fig. 3(b)] [43], whereas it is difficult to prove the fractality rigorously through numerical simulations. At least, the estimated dimension $D_{0}$ suggests the transition from a torus to chaos with a torus occurs via fractalization of the torus (i.e., strange nonchaotic attractor; SNA) [44-46], although frequency lockings to lower-dimensional torus intervened in by parameter $b$ make it harder to confirm this.

In summary, we investigated globally coupled circle maps to understand if and how chaos appears from a highdimensional torus. First, we found that $T^{N}$ exist even for large $N$. Next, despite the prevalence of torus attractors, chaos can appear even in the invertible regime (i.e., for lower nonlinearity compared to the case with $N=1$ ), and its fraction increases with $N$ and is then saturated. In the case of $N \geqslant 3$, chaos in the invertible regime often "coexists with a torus." Such chaos has a number $[O(N)]$ of null Lyapunov exponents. This chaos with a torus has both the faces of torus and chaos: The chaos mode is extended over a (high-dimensional) torus (not localized as in standard chaos), but is not spread equally over all the dimension of the torus. It is characterized by the anomalous decrease of the inverse partition ratio of the first Lyapunov vector, $Y_{2}$, against the torus dimension $M$ (and Lyapunov dimension $D_{L}$ ). Slow itinerant motion of the chaos mode is also observed, suggesting slower changes in the stretching directions.

Indeed, long-term, chaotic change over lower-dimensional states (termed as attractor ruins) is known as chaotic itinerancy, where the accumulation of Lyapunov exponents close to 0 is reported $[47,48]$, similar to chaos with a torus in the present paper. In this respect, chaos with a torus might be interpreted as chaotic itinerancy over lower-dimensional tori on a high-dimensional torus [49].

We also discussed the possible relevance of fractal torus to the transition to chaos with a torus. The existence of fractal torus was investigated as SNA in a forced system [44-46], while no conclusive evidence for its existence is given in an autonomous system thus far. It is often believed that SNA exists not in autonomous systems but only in forced systems; though, in the present case with $N \geqslant 3$, one neutral mode remains for the transition from a torus to chaos with a torus, and that mode might work as quasiperiodic forcing, which may allow for SNA at this transition. Mean-field analysis combining Kuramoto model [5] and collective chaos in globally coupled maps [51] will be an important future problem [52].

Note that the torus dimension is extensive, i.e., the number of null Lyapunov exponents, $M$, increases as $O(N)$ when $N \rightarrow$ $\infty$. In addition, we numerically confirmed that the above results are qualitatively reproduced even when different coupling forms are adopted: e.g., when the coupling constants $J_{i j}$ are heterogeneous (i.e., dependent on $i, j$ ) and randomly chosen, or when the interaction term is given by $f\left(x_{i}(t), x_{j}(t)\right)=$ $\sin \left(2 \pi\left(x_{i}(t)-x_{j}(t)\right)\right)$ like the Kuramoto model [5] (Supplemental Material [30], Sec. S1). Whereas the condition of coupling forms and parameter values for the appearance of chaos with a torus needs to be further explored in the future, the generality of the existence of high-dimensional tori and chaos with a torus is thus suggested. Accordingly, one may expect a variety of possible applications of chaos with a torus. Here, we briefly mention a possible example, albeit they may be speculative at the current stage.

In electroencephalogram (EEG), several peaks with different frequencies exist in its power spectra-alpha, beta, gamma, delta, theta, and mu waves. EEG also involves continuous power spectra besides these peaks, suggesting the existence of rich aperiodic (chaotic) modes, together with quasiperiodicity with different frequencies. Moreover, possible relevance of chaotic itinerancy for autonomously switching internal modes is discussed both theoretically $[31,47,53]$ and experimentally [54-56]. Although the complexity of 
neural dynamics is much higher, chaos with a torus may provide a novel perspective to it.

Finally, we come back to the original problem in this paper, concerning turbulent behavior. As mentioned already, the relevance of high-dimensional tori to it has been discussed in many works since Landau [20] (see, e.g., Ref. [57] for recent proposition); whereas the viewpoint on low-dimensional chaos has been dominant after the proposition by Ruelle and Takens [21]. Probably, both of the two pictures may be necessary to understand the full spectrum of turbulent behavior (not necessarily restricted to fluid turbulence). Chaos with a torus provides an example to integrate the classic viewpoints of Landau's high-dimensional torus and Ruelle and Takens' low-dimensional chaos.
[1] P. Berge, Y. Pomeau, and C. Vidal, The Order in Chaos (Wiley, New York, 1984).

[2] E. Ott, Chaos in Dynamical Systems (Cambridge University Press, Cambridge, 2002).

[3] M. Cencini, F. Cecconi, and A. Vulpiani, Chaos: From Simple Models to Complex Systems (World Scientific, Singapore, 2009).

[4] K. Kaneko, Collapse of Tori and Genesis of Chaos in Dissipative Systems (World Scientific, Singapore, 1986).

[5] Y. Kuramoto, Chemical Oscillations, Waves, and Turbulence (Springer, Berlin, 1984).

[6] J. Stavans, Experimental study of quasiperiodicity in a hydrodynamical system, Phys. Rev. A 35, 4314 (1987).

[7] J. P. Gollub and S. V. Benson, Many routes to turbulent convection, J. Fluid Mech. 100, 449 (1980); A. Libchaber, S. Fauve and C. Laroche, Two-parameter study of the routes to chaos, Physica D 7, 73 (1983).

[8] H. G. Winful, Y. C. Chen, and J. M. Liu, Frequency locking, quasiperiodicity, and chaos in modulated self-pulsing semiconductor lasers, Appl. Phys. Lett. 48, 616 (1986); D. Merbach, O. Hess, H. Herzel, and E. Schöll, Injection-induced bifurcations of transverse spatiotemporal patterns in semiconductor laser arrays, Phys. Rev. E 52, 1571 (1995).

[9] A. Cumming and P. S. Linsay, Quasiperiodicity and Chaos in a System with Three Competing Frequencies, Phys. Rev. Lett. 60, 2719 (1988).

[10] M. R. Bassett and J. L. Hudson, Quasiperiodicity and chaos during an electrochemical reaction, J. Phys. Chem. 93, 2731 (1989); T. Hauck and F. W. Schneider, Mixed-mode and quasiperiodic oscillations in the peroxidase-oxidase reaction, ibid. 97, 391 (1993).

[11] L. Glass and M. C. MacKey, From Clocks to Chaos: The Rhythms of Life (Princeton University Press, Princeton, 1988); L. Glass, Cardiac arrhythmias and circle maps-A classical problem, Chaos 1, 13 (1991); A. Garfinkel, P. S. Chen, D. O. Walter, H. S. Karagueuzian, B. Kogan, S. J. Evans, M. Karpoukhin, C. Hwang, T. Uchida, M. Gotoh, O. Nwasokwa, P. Sager, and J. N. Weiss, Quasiperiodicity and chaos in cardiac fibrillation, J. Clin. Investig. 99, 305 (1997).

[12] A. J. Mandell and K. A. Selz, The Impact of Chaos on Science and Society (United Nations University Press, Tokyo, 1997), pp. 64-96.

[13] J. P. Eckmann, Roads to Turbulence in Dissipative Dynamical Systems, Rev. Mod. Phys. 53, 643 (1981).

[14] M. J. Feigenbaum, The universal metric properties of nonlinear transformations, J. Stat. Phys. 21, 669 (1979).
[15] Y. Pomeau and P. Manneville, Intermittent transition to turbulence in dissipative dynamical systems, Commun. Math. Phys. 74, 189 (1980).

[16] M. J. Feigenbaum, L. P. Kadanoff, and S. J. Shenker, Quasiperiodicity in dissipative systems: a renormalization group analysis, Physica D 5, 370 (1982).

[17] D. Rand, S. Ostlund, J. Sethna, and E. D. Siggia, Universal Transition from Quasiperiodicity to Chaos in Dissipative Systems, Phys. Rev. Lett. 49, 132 (1982).

[18] J. A. Glazier and A. Libchaber, Quasi-periodicity and dynamical systems: An experimentalist's view, IEEE Trans. Circ. Sys. 35, 790 (1988).

[19] K. Kaneko, On the period-adding phenomena at the frequency locking in a one-dimensional mapping, Prog. Theor. Phys. 68, 669 (1982); Similarity structure and scaling property of the period-adding phenomena, 69, 403 (1983).

[20] L. D. Landau, On the problem of turbulence, Dokl. Akad. Nauk. SSSR 44, 339 (1944) [C. R. Acad. Sci. USSR 44, 311 (1944)]; reprinted in L. D. Landau and E. M. Lifshitz, Fluid Mechanics (Pergamon, London, 1959).

[21] D. Ruelle and F. Takens, On the nature of turbulence, Commun. Math. Phys. 20, 167 (1971); Note concerning our paper "On the nature of turbulence", 23, 343 (1971).

[22] S. Newhouse, D. Ruelle, and F. Takens, Occurrence of strange Axiom $A$ attractors near quasi periodic flows on $T^{m}, m \geqslant 3$, Commun. Math. Phys. 64, 35 (1978).

[23] D. Turaev, Maps close to identity and universal maps in the newhouse domain, Commun. Math. Phys. 335, 1235 (2015).

[24] C. Grebogi, E. Ott, and J. A. Yorke, Are Three-Frequency Quasiperiodic Orbits to be Expected in Typical Nonlinear Dynamical Systems? Phys. Rev. Lett. 51, 339 (1983); Attractors on an N-torus: Quasiperiodicity versus chaos, Physica D 15, 354 (1985).

[25] K. Kaneko, Fates of three-Torus I - Double Devil's staircases in lockings, Prog. Theor. Phys. 71, 282 (1984).

[26] C. Baesens, J. Guckenheimer, S. Kim, and R. S. MacKay, Three coupled oscillators: mode-locking, global bifurcations and toroidal chaos, Physica D 49, 387 (1991).

[27] D. Li, A three-scroll chaotic attractor, Phys. Lett. A 372, 387 (2007); C. Letellier and R. Gilmore, Poincaré sections for a new three-dimensional toroidal attractor, J. Phys. A 42, 015101 (2009).

[28] J. H. Curry and J. A. Yorke, The Structure of Attractors in Dynamical Systems (Springer, Berlin, 1978), pp. 48-66.

[29] The last term in Eq. (1) is regarded as 0 with $N=1$.

[30] See Supplemental Material at http://link.aps.org/supplemental/ 10.1103/PhysRevResearch.2.023044 for complete locking, the 
relationships between the torus dimension $M$ and Lyapunov dimension $D_{L}$, and the results with different models and parameters.

[31] K. Kaneko, Clustering, coding, switching, hierarchical ordering, and control in network of chaotic elements, Physica D 41, 137 (1990); Globally coupled circle maps, 54, 5 (1991).

[32] In principle, $M$ null Lyapunov exponents (in a map) do not necessarily imply a torus with $M$ incommensurate frequencies, while the latter leads to $M$ null Lyapunov exponents. The null exponent(s) can appear by the existence of conserved quantit(ies) as in Ref. [33] or at a critical (bifurcation) point such as the Feigenbaum's critical attractor. Our models do not have any conserved quantity. Furthermore, when the coupling terms vanish, it shows $N$-dimensional quasiperiodicity. In addition, attractors with $M$ null Lyapunov exponents are observed with a finite fraction of parameters (rather than only at a critical point). Then, it is natural to consider $M$ null Lyapunov exponents indicate $M$-dimensional quasiperiodicity in our models.

[33] G. M. Mahmoud and M. E. Ahmed, Analysis of chaotic and hyperchaotic conservative complex nonlinear systems, Miskolc Math. Notes 18, 315 (2017).

[34] For $N=2,3,4, a_{c}$ is calculated as $a_{c}(2, b)=1 /(1+$ $b), \quad a_{c}(3, b)=\left(\sqrt{4+4 b^{\prime}-7 b^{\prime 2}}-b^{\prime}\right) /\left(2+2 b^{\prime}-4 b^{\prime 2}\right)$, and $a_{c}(4, b)=\left(\sqrt{1+2 b^{\prime}-2 b^{\prime 2}}-b^{\prime}\right) /\left(1+2 b^{\prime}-3 b^{\prime 2}\right)$, with $b^{\prime} \equiv$ $b /(N-1)$.

[35] We numerically checked that the exponents in this tolerance gradually approach zero as the time steps increase, while the others do not.

[36] This rough estimate also suggests that the fraction of complete locking, $T^{0}$, decreases exponentially with $N$ as $p^{N}$. Accordingly, the decrease of $T^{N}$ and $T^{0}$ can be fitted by $p \simeq 0.36$ for $a=0.7$ and $p \simeq 0.49$ for $a=0.8$ [see also Supplemental Material [30], Fig. S2(a)]. (See also Ref. [37] for Hamiltonian systems.)

[37] M. Falcioni, U. Marini Bettolo Marconi, and A. Vulpiani, Ergodic properties of high-dimensional symplectic maps, Phys. Rev. A 44, 2263 (1991).

[38] In most dynamical systems, scaling in Lyapunov spectra $\hat{\lambda}(x) \equiv$ $\lambda_{N x}(0 \leqslant x \leqslant 1)$ approaches an $N$-invariant form in the $N \rightarrow$ $\infty$ limit $[39,40]$ (see also Fig. 2). Then, for chaos with a torus to exist, $M$ as well as the number of positive Lyapunov exponents should increase in proportion to $N$.

[39] K. Kaneko, Lyapunov analysis and information flow in coupled map lattices, Physica D 23, 436 (1986).

[40] K. A. Takeuchi, F. Ginelli, and H. Chaté, Lyapunov Analysis Captures the Collective Dynamics of Large Chaotic Systems, Phys. Rev. Lett. 103, 154103 (2009).

[41] D. J. Thouless, Electrons in disordered systems and the theory of localization, Phys. Rep. 13, 93 (1974); F. Evers and A. D. Mirlin, Fluctuations of the Inverse Participation Ratio at the Anderson Transition, Phys. Rev. Lett. 84, 3690 (2000).

[42] In the invertible regime, $D_{L} \simeq M+0.3 N$ holds (see Supplemental Material [30], Fig. S3); note there exist $\sim 0.1 N$ positive Lyapunov exponents here. To satisfy $\sum_{j=1}^{\left[D_{L}\right\rceil} \lambda_{j} \leqslant 0$, neg- ative exponents of the same order are needed to cancel the positive sum, which may explain the above relation. In the noninvertible regime, even when $D_{L}$ is large, $M$ is always zero and $Y_{2}$ is nearly 1 .

[43] In the case with $N=2$ where chaos with a torus cannot emerge, torus attractors with noninteger dimensions cannot be found (see Supplemental Material [30], Fig. S7).

[44] C. Grebogi, E. Ott, S. Pelikan, and J. A. Yorke, Strange attractors that are not chaotic, Physica D 13, 261 (1984).

[45] U. Feudel, S. Kuznetsov, and A. Pikovsky, Strange Nonchaotic Attractors: Dynamics Between Order and Chaos in Quasiperiodically Forced Systems (World Scientific, Singapore, 2006).

[46] K. Kaneko, Fractalization of Torus, Prog. Theor. Phys. 71, 1112 (1984); T. Nishikawa and K. Kaneko, Fractalization of a torus as a strange nonchaotic attractor, Phys. Rev. E 54, 6114 (1996).

[47] K. Kaneko and I. Tsuda, Chaos and Beyond: A Constructive Approach With Applications in Life Sciences (Springer, Berlin, 2001); Chaotic Itinerancy, Chaos 13, 926 (2003).

[48] K. Kaneko, Information cascade with marginal stability in network of chaotic elements, Physica D 77, 456 (1994).

[49] Notably, some earlier studies on a chaotic hierarchy claimed chaos with two null Lyapunov exponents [50]. However, one of the "null" exponents does not decay towards zero but rather converges to a small positive value with the increase in time steps, when we ran the simulation much longer time steps.

[50] M. Klein and G. Baier, A Chaotic Hierarchy (World Scientific, Singapore, 1991), pp. 1-24.

[51] T. Shibata and K. Kaneko, Collective Chaos, Phys. Rev. Lett. 81, 4116 (1998); T. Shibata, T. Chawanya, and K. Kaneko, Noiseless Collective Motion out of Noisy Chaos, ibid. 82, 4424 (1999); K. Kaneko, Mean field fluctuation in network of chaotic elements, Physica D 55, 368 (1992).

[52] One could derive a self-consistent equation for the mean field, if it would approach a fixed-point or quasiperiodic solution in the $N \rightarrow \infty$ limit. In contrast, for chaotic motion of each element to persist, such solution is unstable, which makes the mean-field analysis harder [51].

[53] I. Tsuda, Chaotic itinerancy as a dynamical basis of hermeneutics in brain and mind, World Futures 32, 167 (1991).

[54] L. Kay, L. R. Lancaster, and W. J. Freeman, Comparison of EEG time series from rat olfactory system with model composed of nonlinear coupled oscillators, Int. J. Bifur. Chaos 5, 849 (1995).

[55] L. Kay, A challenge to chaotic itinerancy from brain dynamics, Chaos 13, 1057 (2003).

[56] W. J. Freeman, Simulation of chaotic EEG patterns with a dynamic model of the olfactory system, Biol. Cybern. 56, 139 (1987); Evidence from human scalp electroencephalograms of global chaotic itinerancy, Chaos 13, 1067 (2003).

[57] R. Herrero, F. Pi, J. Rius, and G. Orriols, About the oscillatory possibilities of the dynamical systems, Physica D 241, 1358 (2012); R. Herrero, J. Farjas, F. Pi, and G. Orriols, Nonlinear oscillatory mixing in the generalized Landau scenario, Phys. Rev. E 97, 052218 (2018). 